\title{
Demanda hídrica do milho de cultivo de inverno no Estado do Rio de Janeiro
}

Daniel F. de Carvalho ${ }^{1}$, Eleandro S. da Cruz $^{2}$, Wilson A. da Silva ${ }^{3}$, Wanderley de J. Souza ${ }^{4}$ \& Teodorico A. Sobrinho ${ }^{5}$

\section{RESUMO}

O presente trabalho foi desenvolvido com o objetivo de estimar a demanda de irrigação suplementar para a cultura do milho, no Estado do Rio de Janeiro, utilizando-se coeficientes de cultivo (Kc) corrigidos para as diferentes fases de desenvolvimento da cultura. Através de dados climáticos de 34 estações meteorológicas, a demanda de irrigação foi simulada para a cultura do milho cu1tivada no inverno, por meio de um programa computacional. Os valores de Kc corrigidos para o milho variaram de 0,57 a 0,71, para a fase I; de 0,9 a 1,12, para a fase III e de 0,63 a 0,86, para a fase IV. Com esses valores de Kc em comparação com os valores de Kc padrão, obteve-se uma diferença média na lâmina de irrigação de $71,5 \mathrm{~mm}$.

Palavras-chave: coeficiente de cultivo, lâmina de irrigação, Zea mays L.

\section{Water demand of winter corn in the State of Rio de Janeiro, Brazil}

\begin{abstract}
This study was conducted in order to estimate the supplemental corn water demand, in Rio de Janeiro State, Brazil, using corrected crop coefficients $(\mathrm{Kc})$ for different phases of corn development. Using data from 34 meteorological stations, the irrigation demand was simulated for the corn crop for the winter season through computational program. The corrected Kc values for corn varied from 0.57 to 0.71 in the development stage I, from 0.9 to 1.12 in the stage III and from 0.63 to 0.86 in the stage IV in Rio de Janeiro State. Using these corrected Kc values in detriment of standard valu$\mathrm{es}$, an average difference in the irrigation demand of $71.5 \mathrm{~mm}$ in this region was obtained.
\end{abstract}

Key words: crop coefficient, irrigation depth, corn

${ }^{1}$ Departamento de Engenharia/UFRRJ. BR 465, km 7 - Seropédica-RJ. Fone: (21) 3787-3742. E-mail:carvalho@ufrrj.br

${ }^{2}$ Mestrando em Agronomia - Ciência do Solo/UFRRJ. E-mail: el-cruz@bol.com.br

${ }^{3}$ Doutorando em Agronomia - Ciência do Solo/UFFRJ. Fone: 21 3787-4496. wilsonufrrj@yahoo.com.br

${ }^{4}$ Bolsista de IC-PIBIC/UFRRJ/CNPq.

${ }^{5}$ Departamento de Ciências Agrárias/UFMS. CP 533, CEP: 79804-970, Dourados, MS. Fone: (67) 411-3826; E-mail: talves@ceud.ufms.br. 


\section{INTRODUÇÃO}

O milho (Zea mays L.) é uma cultura de amplo aproveitamento, não só por se adaptar às condições edafoclimáticas brasileiras, alcançando elevados níveis de produção, mesmo em condições adversas, mas tambem por representar uma boa fonte protéica. O Brasil é o terceiro maior produtor mundial, com cerca de $7 \%$ da produção, ficando atrás apenas da China e Estados Unidos (Brugnago Neto, 1996).

No Brasil existem distintos modelos de produção de milho, de acordo com a região. Segundo Cogo (1992), nas áreas produtoras nacionais de milho ocorrem duas estações de cultivo no ano agrícola: uma corresponde a 95\% da produção total, no período mais chuvoso, e a outra aos 5\% restantes, denominada plantio extemporâneo ou "safrinha”, plantada em um período mais seco. O plantio de milho na estação de cultivo, denominada "safrinha”, vem ganhando cada vez mais área, cuja viabilidade é, na maioria das vezes, baseada no uso da irrigação suplementar, uma vez que as precipitações pluviométricas que ocorrem durante o desenvolvimento da cultura não são suficientes para suprir sua necessidade hídrica.

Embora o milho plantado fora de época passe por períodos em que a precipitação pluvial não é tão expressiva como no restante do ano (Cardoso et al., 2001) esta, na forma de precipitação efetiva, deve ser contabilizada no cálculo da lâmina de irrigação total por representar uma entrada de água no sistema (Bernardo, 1995; Saad \& Frizzone, 1998; Sousa, 2002).

No dimensionamento de projetos de irrigação o uso de parâmetros adequados às condições locais e da cultura constitui uma importante ferramenta para se alcançar determinada eficiência no uso dos recursos hídricos, sendo a evapotranspiração de referência (ETo) uma variável fundamental na estimativa da demanda de água pela planta (Kashyap \& Panda, 2001). Jensen et al. (1990) definem ETo como sendo a taxa com que a água, se disponível, seria removida da superfície do solo e de uma planta específica, arbitrariamente chamada cultura de referência. É normalmente expressa como a taxa de troca de calor latente por unidade de área ou lâmina de água evaporada. Dentro deste contexto, um parâmetro muito significativo é o coeficiente de cultivo (Kc), razão entre a evapotranspiração potencial da cultura e a evapotranspiração de referência (ETc/ETo) (Doorenbos \& Pruitt, 1977). Este parâmetro está diretamente relacionado à demanda hídrica da cultura, sob determinadas condições climáticas do local e fisiológicas sendo, desta forma, variável no tempo e no espaço. Por isto, é desejável se obter valores de Kc ajustados para as diferentes fases de desenvolvimento da planta e para as diferentes regiões de plantio, possibilitando uma eficiência melhor de uso da água.

A demanda hídrica do milho, como de qualquer outra cultura, é uma função dos fatores climáticos predominantes na região, da variedade e do estádio de desenvolvimento da cultura, do tipo de solo e do sistema de irrigação adotado (Araújo et al., 1999). Esta demanda pode ser obtida pela multiplicação da evapotranspiração de referência (ETo) pelo coeficiente de cultivo (Kc). De acordo com Allen et al. (1998), o modelo de Penman-Monteith proporciona estima- tivas confiáveis e consistentes de ETo. Segundo Smith (1991), este modelo foi considerado o de melhor desempenho entre os métodos combinados, sendo recomendado pela FAO como método padrão para obtenção da ETo, sendo universalmente aceita para estimativas horárias e diárias da ETo (Alexandris \& Kerkides, 2003). O Kc deve ser estimado para as diferentes fases de desenvolvimento (inicial, intermediário e final) e para cada localidade, com base nas condições climáticas locais. Esta determinação pode ser feita por meio de modelos físico-matemáticos propostos por Albuquerque et al. (2001) e por Pereira \& Allen (1997).

Em virtude da importância de se obter informações regionais confiáveis para melhor se dimensionar sistemas hidroagrícolas, desenvolveu-se este trabalho com o objetivo de estimar os coeficientes de cultivo locais para a cultura do milho cultivado no inverno e sua influência na demanda de irrigação para o Estado do Rio de Janeiro.

\section{MATERIAL E MÉTODOS}

Para maior abrangência do estudo, simulou-se o plantio de inverno da cultura do milho no Estado do Rio de Janeiro, localizado na região sudeste do Brasil, entre 20 e $24^{\circ}$ de Latitude Sul e 40 a $45^{\circ}$ de Longitude a Oeste de Greenwich. Para obtenção da demanda de irrigação, foram utilizados dados de 34 estações climatológicas pertencentes à rede do INMET (Instituto Nacional de Meteorologia), das quais 25 se localizavam no Estado do Rio de Janeiro, 2 em São Paulo, 6 em Minas Gerais e 1 no Espírito Santo. Essas estações possuem altitudes que variaram desde o nível do mar até aproximadamente 960 m. A seleção de estações fora do Estado do Rio de Janeiro se deu para fins de melhoria na interpolação dos dados.

A seleção das estações foi baseada na disponibilidade de dados climáticos diários e na sua distribuição espacial, sendo adotadas séries históricas com dados diários cujo período base variou de 1961 a 1978. Na Tabela 1 estão apresentadas as estações utilizadas e, na Figura 1, visualiza-se a distribuição espacial das mesmas.

No Estado do Rio de Janeiro, sobretudo no norte fluminense tradicionalmente, após a colheita do arroz no final de abril, as várzeas ficam ociosas durante o período de maio a outubro, quando a rebrota do arroz e a vegetação espontânea são utilizadas como pastagem, ou então se planta o milho de inverno (Souza Filho et al., 1987); sendo assim, o plantio do milho foi simulado para o mês de maio e se considerou um ciclo da cultura de 150 dias distribuídos nas quatro fases de desenvolvimento (Doorenbos \& Pruitt, 1977). A Tabela 2 apresenta a distribuição das fases com suas respectivas datas.

Ressalta-se que, apesar da metodologia baseada em graus dias ser a mais adequada para se estabelecer a duração das fases de desenvolvimento, sobretudo para a cultura do milho, esta não foi utilizada em virtude da sensível variação da temperatura no Estado do Rio de Janeiro, o que dificultaria a definição de um período comum de duração das fases para todas as estações. 
Tabela 1. Código, nome, latitude e longitude e altitude das estações utilizadas

\begin{tabular}{|c|c|c|c|c|}
\hline Código & Nome & Latitude Sul & $\begin{array}{l}\text { Longitude } \\
\text { Oeste }\end{array}$ & $\begin{array}{c}\text { Altitude } \\
\text { (m) }\end{array}$ \\
\hline 83697 & São Fidelis - RJ & $21^{\circ} 40^{\prime} 25^{\prime \prime}$ & $41^{\circ} 45^{\prime} 24^{\prime \prime}$ & 74,0 \\
\hline 83698 & Campos - RJ & $21^{\circ} 45^{\prime} 00^{\prime \prime}$ & $41^{\circ} 19^{\prime} 59^{\prime \prime}$ & 11,0 \\
\hline 83695 & Itaperuna - RJ & $21^{\circ} 12^{\prime} 00^{\prime \prime}$ & $41^{\circ} 54^{\prime} 00^{\prime \prime}$ & 124,0 \\
\hline 83805 & Santo Antônio de Pádua - RJ & $21^{\circ} 32^{\prime} 32^{\prime \prime}$ & $42^{\circ} 10^{\prime} 50^{\prime \prime}$ & 96,0 \\
\hline 83696 & Santa Maria Madalena - RJ & $21^{\circ} 54^{\prime} 00^{\prime \prime}$ & $42^{\circ} 01^{\prime} 00^{\prime \prime}$ & 620,0 \\
\hline 83807 & Carmo - RJ & $21^{\circ} 26^{\prime} 00^{\prime \prime}$ & $42^{\circ} 37^{\prime} 00^{\prime \prime}$ & 341,0 \\
\hline 83749 & Macaé - RJ & $22^{\circ} 22^{\prime} 59^{\prime \prime}$ & $41^{\circ} 48^{\prime} 00^{\prime \prime}$ & 4,0 \\
\hline 83747 & Cabo Frio - RJ & $22^{\circ} 52^{\prime} 59^{\prime \prime}$ & $42^{\circ} 01^{\prime} 00^{\prime \prime}$ & 3,0 \\
\hline 83745 & Nova Friburgo - RJ & $22^{\circ} 17^{\prime} 00^{\prime \prime}$ & $42^{\circ} 31^{\prime} 59^{\prime \prime}$ & 857,0 \\
\hline 83806 & Teresópolis (Parque Nacional) - RJ & $22^{\circ} 25^{\prime} 59^{\prime \prime}$ & $42^{\circ} 58^{\prime} 59^{\prime \prime}$ & 959,0 \\
\hline 83718 & Cordeiro - RJ & $22^{\circ} 01^{\prime} 59^{\prime \prime}$ & $42^{\circ} 22^{\prime} 59^{\prime \prime}$ & 485,0 \\
\hline 83790 & Bangu - RJ & $22^{\circ} 52^{\prime} 00^{\prime \prime}$ & $43^{\circ} 27^{\prime} 00^{\prime \prime}$ & 40,0 \\
\hline 83742 & Vassouras - RJ & $22^{\circ} 25^{\prime} 00^{\prime \prime}$ & $43^{\circ} 40^{\prime} 00^{\prime \prime}$ & 437,0 \\
\hline 83763 & Tinguá - RJ & $22^{\circ} 34^{\prime} 59^{\prime \prime}$ & $43^{\circ} 21^{\prime} 00^{\prime \prime}$ & 125,0 \\
\hline 83764 & Xerém - RJ & $22^{\circ} 34^{\prime} 59^{\prime \prime}$ & $43^{\circ} 18^{\prime} 00^{\prime \prime}$ & 144,0 \\
\hline 83759 & São Pedro da Aldeia - RJ & $22^{\circ} 51^{\prime} 21^{\prime \prime}$ & $42^{\circ} 07^{\prime} 09^{\prime \prime}$ & 178,0 \\
\hline 83757 & Piraí - RJ & $22^{\circ} 38^{\prime} 00^{\prime \prime}$ & $43^{\circ} 52^{\prime} 59^{\prime \prime}$ & 388,0 \\
\hline 83789 & Santa Cruz - RJ & $22^{\circ} 53^{\prime} 18^{\prime \prime}$ & $43^{\circ} 44^{\prime} 16^{\prime \prime}$ & 63,0 \\
\hline 83741 & Ecologia Agrícola (Km 47) - RJ & $22^{\circ} 46^{\prime} 00^{\prime \prime}$ & $43^{\circ} 40^{\prime} 59^{\prime \prime}$ & 33,0 \\
\hline 83765 & Araras - RJ & $22^{\circ} 31^{\prime} 00^{\prime \prime}$ & $43^{\circ} 10^{\prime} 59^{\prime \prime}$ & 820,0 \\
\hline 83762 & Rio D'ouro - RJ & $22^{\circ} 37^{\prime} 00^{\prime \prime}$ & $43^{\circ} 28^{\prime} 00^{\prime \prime}$ & 128,0 \\
\hline 83752 & Barreirinha - RJ & $22^{\circ} 24^{\prime} 57^{\prime \prime}$ & $44^{\circ} 43^{\prime} 34^{\prime \prime}$ & 757,0 \\
\hline 83753 & Volta Redonda - RJ & $22^{\circ} 29^{\prime} 00^{\prime \prime}$ & $44^{\circ} 04^{\prime} 59^{\prime \prime}$ & 418,0 \\
\hline 83738 & Resende - RJ & $22^{\circ} 29^{\prime} 00^{\prime \prime}$ & $44^{\circ} 26^{\prime} 43^{\prime \prime}$ & 440,0 \\
\hline 83788 & Angra dos Reis - RJ & $22^{\circ} 59^{\prime} 55^{\prime \prime}$ & $44^{\circ} 18^{\prime} 25^{\prime \prime}$ & 2,0 \\
\hline 83646 & Cachoeiro do Itapemirim - ES & $20^{\circ} 51^{\prime} 00^{\prime \prime}$ & $41^{\circ} 06^{\prime} 00^{\prime \prime}$ & 78,0 \\
\hline 83686 & Caxambu - MG & $21^{\circ} 59^{\prime} 00^{\prime \prime}$ & $44^{\circ} 57^{\prime} 00^{\prime \prime}$ & 959,0 \\
\hline 83690 & Santos Dumont - MG & $21^{\circ} 27^{\prime} 00^{\prime \prime}$ & $43^{\circ} 33^{\prime} 00^{\prime \prime}$ & 908,0 \\
\hline 83690 & Muriaé - MG & $21^{\circ} 08^{\prime} 00^{\prime \prime}$ & $42^{\circ} 19^{\prime} 59^{\prime \prime}$ & 271,0 \\
\hline 83711 & Núcleo Colonial Bandeirantes - SP & $22^{\circ} 36^{\prime} 00^{\prime \prime}$ & $44^{\circ} 28^{\prime} 00^{\prime \prime}$ & 545,0 \\
\hline 83712 & Bananal - SP & $22^{\circ} 42^{\prime} 00^{\prime \prime}$ & $44^{\circ} 19^{\prime} 00^{\prime \prime}$ & 560,0 \\
\hline 83737 & Passa Quatro - MG & $22^{\circ} 23^{\prime} 00^{\prime \prime}$ & $44^{\circ} 58^{\prime} 00^{\prime \prime}$ & 920,0 \\
\hline 83027 & Cataguases - MG & $21^{\circ} 23^{\prime} 00^{\prime \prime}$ & $42^{\circ} 41^{\prime} 00^{\prime \prime}$ & 168,0 \\
\hline 83037 & Coronel Pacheco (Água Limpa) - MG & $21^{\circ} 55^{\prime} 00^{\prime \prime}$ & $43^{\circ} 15^{\prime} 00^{\prime \prime}$ & 435,0 \\
\hline
\end{tabular}

Tabela 2. Datas utilizadas nas simulações das fases do ciclo da cultura do milho

\begin{tabular}{lc}
\hline \multicolumn{1}{c}{ Fases do ciclo } & Período \\
I - Plantio até estabelecimento da cultura & $01 / 05$ a 30/05 \\
II - Crescimento vegetativo & $31 / 05$ a 09/07 \\
III - Floração & $10 / 07$ a 28/08 \\
IV - Enchimento dos grãos até maturação & $29 / 08$ a 27/09 \\
\hline
\end{tabular}

Fonte: Doorenbos \& Pruitt (1977)

Para estimativa dos coeficientes de cultivo inicial, intermediário e final, foram utilizadas as equações 1, 2 e 3, respectivamente, em que a equação 1 foi proposta por Albuquerque et al. (2001) e as equações 2 e 3, por Pereira \& Allen (1997).

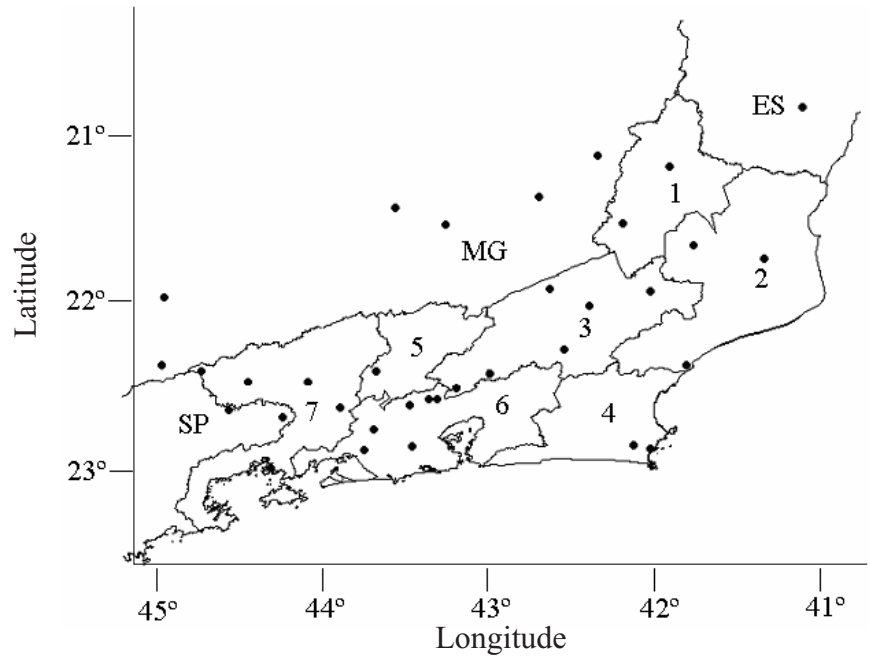

Figura 1. Estado do Rio de Janeiro subdividido em mesorregiões (1: Noroeste; 2: Norte; 3: Serrana; 4: Lagos; 5: Centro Sul; 6: Metropolitana; 7: Sul), Espírito Santo, Minas Gerais e São Paulo, e as estações climatológicas pertencentes à rede do INMET utilizadas no estudo

$$
\begin{gathered}
\mathrm{Kc}_{\mathrm{i}}=1,42-0,09 \cdot \mathrm{ETo}-0,11 \cdot \mathrm{IE}+0,004 \cdot \mathrm{ETo}^{2}+0,003 \cdot \mathrm{IE}^{2} \\
+ \text { 0,0003.ETo } \mathrm{IE}
\end{gathered}
$$

$$
\begin{aligned}
& \mathrm{Kc}_{\mathrm{m}}=\mathrm{Kc}_{\mathrm{m}}(\text { Padrão })+\left[0,04\left(\mathrm{U}_{2}-2\right)-0,004\left(\mathrm{UR}_{\text {mín }}-45\right)\right] \cdot\left(\frac{\mathrm{h}_{1}}{3}\right)^{0,3} \\
& \mathrm{Kc}_{\mathrm{f}}=\mathrm{Kc}_{\mathrm{f}}(\text { Padrão })+\left[0,04\left(\mathrm{U}_{2}-2\right)-0,004\left(\mathrm{UR}_{\text {mín }}-45\right)\right] \cdot\left(\frac{\mathrm{h}_{2}}{3}\right)^{0,3}
\end{aligned}
$$

em que:

$\mathrm{Kc}_{\mathrm{m}}$ (Padrão) - Coeficiente de cultura médio recomendado segundo Doorenbos \& Pruitt (1977)

$\mathrm{Kc}_{\mathrm{f}}$ (Padrão) - Coeficiente de cultura final recomendado segundo Doorenbos \& Pruitt (1977)

ETo - Evapotranspiração de referência na fase 1 (mm.dia $\left.{ }^{-1}\right)$

IE - Intervalo entre eventos de umedecimento do solo (chuva ou irrigação em dias)

$\mathrm{U}_{2}$ - Velocidade dos ventos a $2 \mathrm{~m}$ de altura

$\mathrm{UR}_{\text {mín }}$ - Umidade relativa mínima

$\mathrm{h}_{1}$ - Altura média da cultura na fase 3

$\mathrm{h}_{2} \quad$ - Altura média da cultura na fase 4

Os valores de ETo utilizados na equação foram estimados por Carvalho et al. (2001), utilizando o modelo PenmanMontheith. Obtiveram-se os demais parâmetros $\left(\mathrm{U}_{2}\right.$ e $\left.\mathrm{UR}_{\min }\right)$ levando-se em consideração o número de dias correspondentes a cada uma das fases de desenvolvimento da cultura (Tabela 2). Os valores de $\mathrm{Kc}_{\mathrm{m}}$ (Padrão) e $\mathrm{Kc}_{\mathrm{f}}$ (Padrão), foram 1,05 e 0,80 , respectivamente, segundo Doorenbos \& Pruitt (1977). A altura das plantas nas fases III e IV foi de $2 \mathrm{~m}$ e o intervalo entre eventos de umedecimento adotado foi de 7 dias, uma vez que na região norte-fluminense é crescente o uso de sistemas de irrigação por aspersão, para os quais usualmente se adota este período como turno de rega (Sousa et al., 2002), sendo este método empregado em cerca de $60 \%$ dos empreendimentos agrícolas em que se faz uso de irrigação nesta região (Azevedo, 1998). 
De posse dos valores de Kc corrigidos para o milho em cada estação climatológica utilizada, foram gerados mapas temáticos, cujos coeficientes foram espacializados através do software IDRISI 32 (Eastman, 1999); em seguida, com os valores de Kc corrigido e padrão procedeu-se, para cada estação, ao cálculo das demandas de irrigação suplementar através do programa computacional DEMANDA (Carvalho et al., 1999), tendo em vista o plantio da cultura em um solo com disponibilidade total de água de 0,6 mm por cm de solo.

Para a estimativa da lâmina de precipitação dependente, necessária para o cálculo da demanda de irrigação suplementar, utilizou-se a distribuição gama, a nível de 75\% de probabilidade, em função das alturas médias pentadiais dos dias chuvosos, conforme Sediyama et al. (1996). Os valores da precipitação dependente totalizados por fase de desenvolvimento são apresentados na Tabela 3. Nota-se que, para as

Tabela 3. Valores de lâminas de precipitação dependente $(\mathrm{mm})$ para cada estação, nas diferentes fases de desenvolvimento da cultura do milho

\begin{tabular}{|ccccc|}
\hline Código & Fase I & Fase II & Fase III & Fase IV \\
\hline 83697 & 37,5 & 49,7 & 60,7 & 45,5 \\
\hline 83698 & 14,5 & 14,9 & 30,0 & 17,2 \\
\hline 83695 & 9,0 & 10,8 & 21,8 & 21,0 \\
\hline 83805 & 23,5 & 29,1 & 37,2 & 36,0 \\
\hline 83696 & 24,5 & 20,9 & 23,1 & 18,8 \\
\hline 83807 & 32,5 & 34,9 & 48,8 & 51,0 \\
\hline 83749 & 23,0 & 26,6 & 29,4 & 23,2 \\
\hline 83747 & 28,5 & 33,9 & 47,5 & 23,1 \\
\hline 83745 & 16,5 & 14,5 & 16,1 & 17,5 \\
\hline 83806 & 40,5 & 27,3 & 38,3 & 43,8 \\
\hline 83718 & 8,0 & 16,4 & 12,9 & 22,2 \\
\hline 83790 & 26,0 & 16,5 & 28,6 & 21,0 \\
\hline 83742 & 10,0 & 13,5 & 10,3 & 14,3 \\
\hline 83763 & 32,5 & 28,6 & 45,4 & 34,4 \\
\hline 83764 & 32,0 & 41,2 & 55,5 & 32,8 \\
\hline 83759 & 43,5 & 50,9 & 44,3 & 40,5 \\
\hline 83757 & 13,5 & 17,1 & 21,5 & 19,8 \\
\hline 83789 & 33,0 & 28,1 & 38,9 & 24,8 \\
\hline 83741 & 23,5 & 18,9 & 19,8 & 19,1 \\
\hline 83765 & 36,5 & 33,7 & 35,6 & 32,8 \\
\hline 83762 & 38,5 & 39,5 & 72,4 & 56,4 \\
\hline 83752 & 13,0 & 20,9 & 17,0 & 17,8 \\
\hline 83753 & 18,5 & 20,8 & 11,3 & 10,7 \\
\hline 83738 & 8,0 & 5,4 & 8,7 & 18,6 \\
\hline 83788 & 33,5 & 45,3 & 40,0 & 34,1 \\
\hline 83646 & 19,0 & 19,3 & 28,1 & 18,6 \\
\hline 83686 & 19,5 & 21,6 & 20,0 & 50,1 \\
\hline 83690 & 59,0 & 4,8 & 8,9 & 13,1 \\
\hline 83690 & 13,0 & 21,6 & 28,4 & 46,0 \\
\hline 83711 & 16,0 & 39,9 & 16,8 & 4,1 \\
\hline 83712 & 34,5 & 40,8 & 36,7 & 37,1 \\
\hline 83737 & 11,5 & 8,5 & 15,9 & 17,0 \\
\hline 83027 & 14,0 & 38,5 & 25,9 & 23,5 \\
\hline 83037 & 17,5 & 35,2 & 22,7 & 28,3 \\
\hline Média & 24,2 & 26,2 & 30,0 & 27,5 \\
\hline Desvio & 12,1 & 12,5 & 15,6 & 12,9 \\
\hline Erro & 49,8 & 47,7 & 52,0 & 46,9 \\
\hline & 2,1 & 2,1 & 2,7 & 2,2 \\
\hline & & & & \\
\hline
\end{tabular}

quatro fases de desenvolvimento da cultura, o coeficiente de variação das precipitações é alto, indicando uma grande variabilidade do regime pluviométrico no Estado.

O cálculo da demanda de irrigação foi então realizado por meio do balanço de água no solo (equação 4), tomando-se como referência a máxima capacidade de armazenamento de água no solo.

$$
\mathrm{Da}_{\mathrm{i}}=\mathrm{Da}_{\mathrm{i}-1}+\left(\mathrm{ETrc}_{\mathrm{i}}+\mathrm{Pp}_{\mathrm{i}}\right)-\left(\mathrm{Pd}_{\mathrm{i}}+\mathrm{I}_{\mathrm{i}}\right)
$$

em que:

$\mathrm{Da}_{\mathrm{i}}$ - lâmina de água armazenada no solo no período i, mm

$\mathrm{Da}_{\mathrm{i}-1}$ - lâmina de água armazenada no solo no período anterior a i, mm

$\mathrm{ETrc}_{\mathrm{i}}$ - evapotranspiração real da cultura no período i, mm

$\mathrm{Pp}_{\mathrm{i}}$ - perda por percolação no período i, mm

$\mathrm{Pd}_{\mathrm{i}}$ - precipitação dependente no período i, mm e

$\mathrm{I}_{\mathrm{i}}$ - lâmina de irrigação aplicada no período i, mm

As estimativas de ETrc foram realizadas multiplicandose a ETo pelos coeficientes de cultura para cada fase de desenvolvimento e pelo coeficiente de umidade do solo (Ks), apresentado na equação 5 (Bernardo, 1995).

$$
\mathrm{Ks}=\frac{\operatorname{Ln}((\mathrm{Ua}-\mathrm{Pm})+1,0)}{\operatorname{Ln}((\mathrm{Cc}-\mathrm{Pm})+1,0)}
$$

em que "Ua” é a umidade atual do solo, em mm.

Feito este procedimento para cada estação, as demandas obtidas foram também espacializadas utilizando-se o software IDRISI 32; depois, com o módulo image calculator do software IDRISI 32, realizou-se uma operação com os mapas gerados a fim de se obter um mapa final contendo a diferença, em mm, entre as demandas de irrigação suplementar no Estado do Rio de Janeiro, considerando-se o uso de coeficiente corrigido e padrão.

Todos os mapas foram gerados com tamanho de célula (pixel) de $500 \mathrm{~m}$ por $500 \mathrm{~m}$, obedecendo-se ao método de interpolação inverso da potência da distância (IDW), com expoente 4 para a distância (Faria et al., 2002).

Após a confecção dos mapas, realizou-se a reclassificação dos valores obtidos em intervalos de classe, em função da amplitude de variação desses valores. Para os mapas referentes aos coeficientes de cultivo corrigidos para as fases intermediária e final, o intervalo de classe foi de 0,04 e, para a fase inicial, o intervalo foi de 0,02 .

\section{RESULTADOS E DISCUSSÃO}

Na Tabela 4 estão apresentados a média, o desvio padrão e o coeficiente de variação dos valores de Kc estimados para o Estado do Rio de Janeiro. Nota-se que os valores de Kc variaram pouco em relação à média, fato evidenciado pelos baixos valores de desvio padrão; no entanto, o Kc final apresentou valores de desvio padrão e coeficiente de variação 
Tabela 4. Média, desvio padrão (DP) e coeficiente de variação (CV) dos valores de Kc inicial, médio e final para o milho no Estado do Rio de Janeiro

\begin{tabular}{cccc}
\hline Medidas estatísticas & Kc inicial & Kc médio & Kc final \\
\hline Média & 0,63 & 0,97 & 0,71 \\
DP & 0,02 & 0,05 & 0,09 \\
CV(\%) & 3,91 & 4,81 & 12,32 \\
\hline
\end{tabular}

Tabela 5. Porcentagens de área do Estado ocupada com as classes de Kc corrigido para o milho

\begin{tabular}{cccccc}
\hline Kc inicial & $\begin{array}{c}\text { Área } \\
\text { ocupada (\%) }\end{array}$ & Kc médio & $\begin{array}{c}\text { Área } \\
\text { ocupada (\%) }\end{array}$ & Kc final & $\begin{array}{c}\text { Área } \\
\text { ocupada (\%) }\end{array}$ \\
\hline $0,57-0,59$ & 2,93 & $0,90-0,94$ & 25,73 & $0,63-0,67$ & 10,21 \\
\hline $0,59-0,61$ & 4,45 & $0,94-0,98$ & 34,57 & $0,67-0,71$ & 30,91 \\
\hline $0,61-0,63$ & 40,27 & $0,98-1,02$ & 35,27 & $0,71-0,75$ & 34,46 \\
\hline $0,63-0,65$ & 30,84 & $1,02-1,06$ & 3,19 & $0,75-0,79$ & 22,34 \\
\hline $0,65-0,67$ & 19,65 & $1,06-1,10$ & 0,71 & $0,79-0,83$ & 1,20 \\
\hline $0,67-0,71$ & 1,86 & $1,10-1,12$ & 0,53 & $0,83-0,86$ & 0,88 \\
\hline Total (\%) & 100,00 & Total (\%) & 100,00 & Total (\%) & 100,00 \\
\hline
\end{tabular}

maiores que os demais, o que pode ser influenciado pelo fato de, nesta situação, a fase IV de desenvolvimento do milho se estender de agosto a setembro, época em que se observa aumento de umidade relativa e velocidade dos ventos no Estado do Rio de Janeiro, influenciados principalmente pela mudança da estação do ano.

Na Figura 2 são apresentados os coeficientes de cultivo corrigidos para o milho nas fases inicial (Figura 2A), intermediária (Figura 2B) e final (Figura 2C). As porcentagens de área do Estado ocupadas com os intervalos de valores desses coeficientes, são apresentadas na Tabela 5 .

Observa-se, na Figura 2A, que os valores de Kc inicial corrigidos oscilaram de 0,57 a 0,71 , sendo que a maior porção do Estado, cerca de 71\%, apresenta valores de Kc inicial variando de 0,61 até 0,65 , sobretudo nas regiões centrosul e sul do Estado. Por outro lado, os menores valores de Kc inicial ocorreram em regiões mais próximas ao mar, mais especificamente nas regiões metropolitana e dos lagos. Neste resultado pode estar a influência da alta umidade relativa do ar, que faz com que a ETo seja menor, diminuindo então os valores de Kc inicial.

Lima et al. (1992) encontraram valor de Kc inicial para o milho, de 0,26, no Estado do Piauí e Andrade Júnior et al. (1998) constataram 0,50 para o mesmo valor no mesmo Estado, evidenciando que este parâmetro é fortemente influenciado por fatores como manejo agrícola, cultivar e condições climáticas. Vê-se que os valores aqui estimados são maiores que os observados pelos autores supracitados, mostrando o efeito da época de plantio na demanda hídrica. Segundo Fancelli \& Dourado Neto (2000), os valores de Kc inicial recomendados para o milho no Brasil, vão de 0,20 a 0,40, porém com base nos resultados aqui obtidos, se esses valores fossem adotados para as condições deste trabalho, a cultura passaria por um estresse, por falta de água no início do
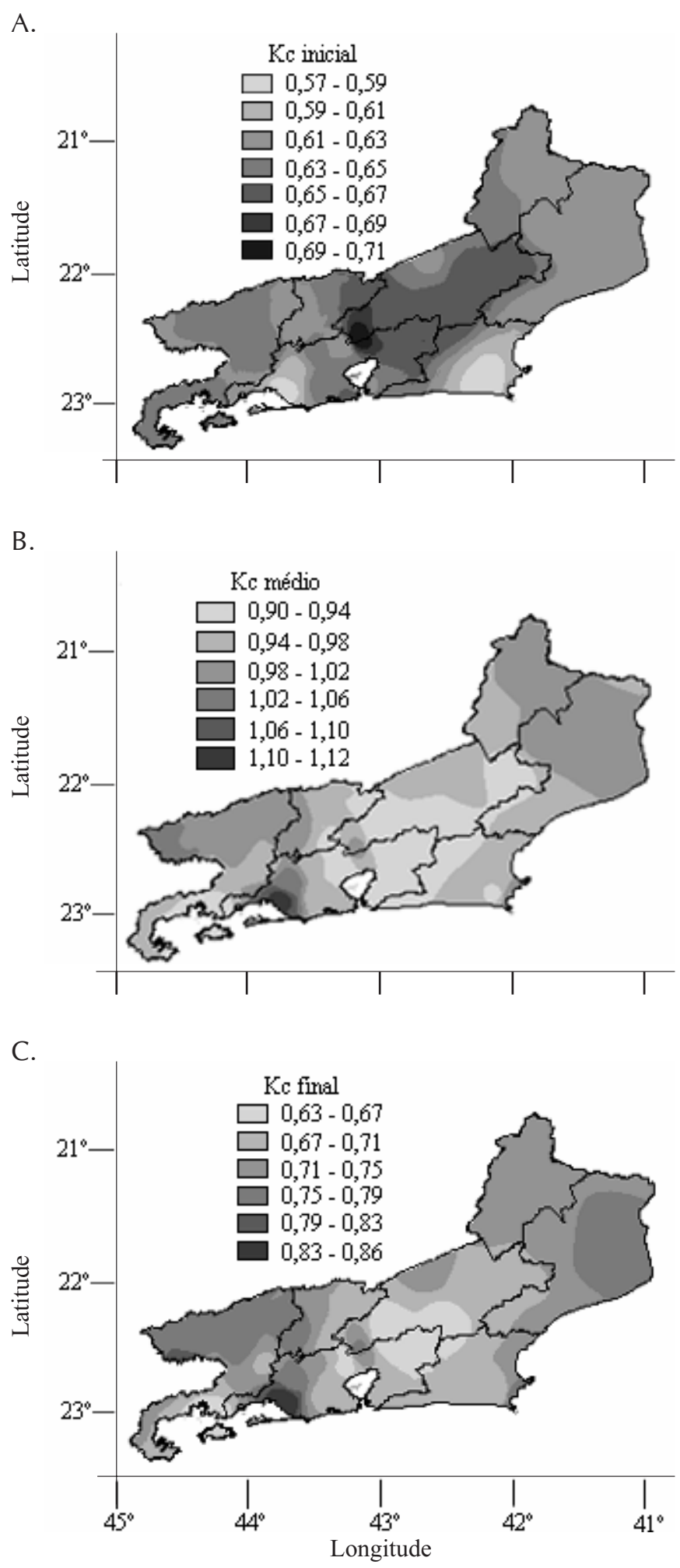

Figura 2. Mapas com os valores corrigidos de Kc inicial (A), médio (B) e final $(\mathrm{C})$, para o milho, no Estado do Rio de Janeiro

desenvolvimento, que é a fase em que a cultura não está com seu sistema radicular totalmente desenvolvido, sendo mais susceptível a competição por água e nutriente, podendo haver, então, reflexo na produção final, porém os valores estimados estão de acordo com a recomendação de Resende et al. (2000), variando na faixa de 0,15 a 1 para o Kc inicial. 
Pode-se observar, ainda na Tabela 5, com auxílio da Figura 2B, que os valores de Kc médio variaram de 0,9 a 1,12 , sendo que os valores mais freqüentes foram de 0,9 a 1,02 , ocupando aproximadamente 95\% da área do Estado, distribuídas por três classes. Os menores valores foram observados na parte central do Estado e em uma parte do sul. Por outro lado, os maiores valores se limitaram a uma pequena área na região metropolitana e em uma parte do norte fluminense. Os referidos valores estão bem próximos dos observados por Lima et al. (1992), e também de acordo com os valores observados por Andrade Júnior et al. (1998), para o Estado do Piauí. Esses valores estão, também, de acordo com os recomendados por Fancelli \& Dourado Neto (2000) e Albuquerque \& Andrade (2000) para as diferentes regiões do Brasil.

De acordo com a Tabela 5 e Figura 2C, os valores de Kc final variaram de 0,63 a 0,86 , sendo que o intervalo mais freqüente foi de 0,67 a 0,79 , ocupando aproximadamente 88\% da área. Os menores valores predominaram na região centro-sul e sul do Estado, enquanto os maiores valores foram observados em uma pequena área na região metropolitana e nas regiões norte e noroeste do Estado. O Kc final indicou uma distribuição semelhante à do Kc médio, e ambos apresentaram distribuição diferente do Kc inicial dentro do Estado. Esses valores foram menores que os observados por Lima et al. (1992) e Andrade Júnior et al. (1998), e maiores que o recomendado por Fancelli \& Dourado Neto (2000) e Albuquerque \& Andrade (2000) para o Brasil.

A Figura 3 apresenta a diferença de demanda de irrigação suplementar para o milho utilizando Kc padrão e Kc corrigido no Estado do Rio de Janeiro. De acordo com a grande variabilidade pluviométrica no Estado (Tabela 3) nota-se, nesta Figura que a diferença na demanda de irrigação suplementar chegou a 103 mm, em uma pequena fração de área do Estado (0,56\%), conforme dados da Tabela 6. Esta área se localiza na região serrana, onde altos índices pluviométricos são observados (Carvalho et al., 2003); em contrapartida, as diferenças de demanda mais recorrentes oscilaram de 40 a $70 \mathrm{~mm}$ e ocupando, juntas, cerca de $85,5 \%$

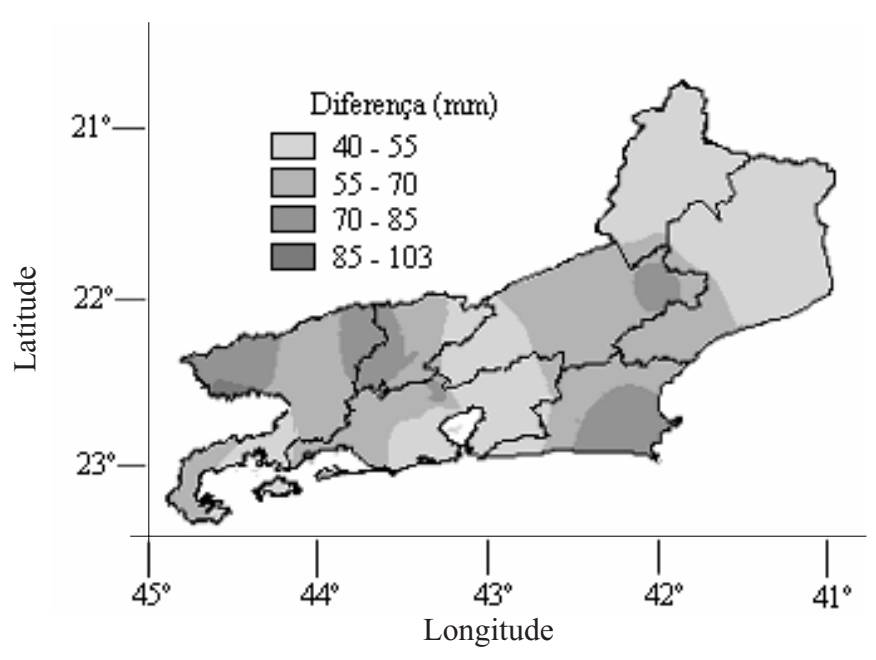

Figura 3. Diferença na demanda total de irrigação suplementar, em mm, utilizando-se Kc padrão e Kc corrigido
Tabela 6. Área do Estado do Rio de Janeiro, em porcentagem, ocupada com as classes de diferença de lâmina de irrigação suplementar para 0 milho, em mm

\begin{tabular}{cc}
\hline Diferença $(\mathbf{m m})$ & \% da Área do Estado \\
$40-55$ & 45,39 \\
$55-70$ & 40,17 \\
$70-85$ & 13,88 \\
$85-103$ & 0,56 \\
Total (\%) & 100 \\
\hline
\end{tabular}

da área do Estado do Rio de Janeiro. As discussões não se basearam em trabalhos realizados na mesma região de estudo, devido ao fato de não terem sido encontrados, na literatura, trabalhos sobre o assunto para o Estado.

A diferença média da demanda de irrigação suplementar no Estado do Rio de Janeiro, utilizando-se coeficientes de cultivo corrigidos e padrão, foi de $71,5 \mathrm{~mm}$ no ciclo da cultura. Considerando-se que o uso da água na irrigação se caracteriza por um intenso uso consuntivo e que as técnicas de manejo da água na agricultura ainda são pouco utilizadas e pouco eficientes, metodologias, como a apresentada neste trabalho, devem ser adotadas em nível de manejo, o que certamente contribuirá para uma utilização mais racional deste recurso.

\section{CONCLUSÕES}

1. Utilizando-se as equações propostas por Albuquerque et al. (2001) e Pereira \& Allen (1997), encontraram-se valores de Kc corrigidos para o milho variando de 0,57 a 0,71 ; 0,90 a 1,12; e 0,63 a 0,86, para as fases inicial, média e final, respectivamente, no Estado do Rio de Janeiro.

2. A economia de lâmina de irrigação suplementar para a cultura do milho cultivado no inverno, no Estado do Rio de Janeiro, proporcionada pela correção do Kc, chegou a 103 mm.

\section{LITERATURA CITADA}

Albuquerque, P. E. P.; Andrade, C. L. T. Uso de planilha eletrônica para a programação da irrigação na cultura do milho. Sete Lagoas: Embrapa Milho e Sorgo, 2000. 24p. Circular Técnica, 5

Albuquerque, P. E. P.; Andrade Junior, A. S.; Sousa, F.; Sediyama, G. C.; Bezerra, J. R. C.; Stone, L. F.; Silveira, P. M. Coeficientes de cultivo das principais culturas anuais. Revista ITEM - Irrigação e Tecnologia Moderna, Brasília, n.52/53, p.49-57. 2001.

Alexandris, S.; Kerkides, P. New empirical formula for hourly estimations of reference evapotranspiration. Agricultural Water Management. Amsterdam. v.60. p.157-180, 2003.

Allen, R. G.; Pereira, L. S.; Raes, D., Smith, M. Crop evapotranspiration: guidelines for computing crop water requirements. Rome: FAO, 1998. 300p. Irrigation and Drainage Paper, 56

Andrade Júnior, A. S.; Cardoso, M. J.; Melo, F. B. Irrigação. In. A cultura do milho no Piauí. 2.ed. Teresina: Embrapa MeioNorte, 1998. p.68-100. Circular Técnica, 12

R. Bras. Eng. Agríc. Ambiental, v.10, n.1, p.112-118, 2006. 
Araújo, W. F.; Sampaio, R. A.; Medeiros, R. D. Irrigação e adubação nitrogenada em milho. Scientia Agrícola, Piracicaba. v.56, n.4. p.909-914. 1999.

Azevedo, H. J. Influência de elementos climáticos no desempenho de sistemas de irrigação por aspersão na região Norte Fluminense. Viçosa: UFV, 1998. 145p. Tese Doutorado

Bernardo, S. Manual de irrigação. 6.ed. Viçosa: UFV, 1995. 657p.

Brugnago Neto, S. Estudo de economia e mercado de produtos agrícolas, 4 - Milho. Instituto de Planejamento e Economia Agrícola de Santa Catarina. Florianópolis: Instituto CEPA/SC, 1996. 72p.

Cardoso, C. O.; Faria, R. T.; Folegatti, M. V. Análise de estratégias de irrigação para o milho "safrinha” em Londrina, com modelos de simulação. Engenharia Rural, Piracicaba, v.12. n. único. p.53-63. 2001.

Carvalho, D. F.; Cruz, E. S.; Guimarães, E. C.; Ceddia, M. B.; Gomes, B. M. Precipitação média mensal do semestre outubro-março para o Estado do Rio de Janeiro, utilizando métodos geoestatísticos. In. Congresso Brasileiro de Engenharia Agrícola 32, 2003, Goiânia. Anais... Goiânia: SBEA-2003, CD Rom.

Carvalho, D. F.; Marques, D. M.; Montebeller, C. A.; Barbosa, L. G.; Santana, G. C. Demanda máxima de irrigação para o milho e o feijão, no Estado do Rio de Janeiro, Brasil. Engenharia Rural, Piracicaba. v.12, n.1, p.21-27. 2001.

Carvalho, D. F.; Soares, A. A.; Sediyama, G. C. Programa computacional para a estimativa da lâmina de irrigação em perímetros irrigados. Engenharia Rural, Piracicaba. v.10, n.2, p.59-73. 1999.

Cogo, C. H. Perspectivas e tendências para o milho na região Sul e no Brasil. In: Congresso Nacional de Milho e Sorgo, 19, 1992. Porto Alegre. Conferências. Porto Alegre, SAA SCT ABMS, 1992. p.71-80.

Doorenbos, J.; Pruitt, W. O. Crop water requirements. Rome: FAO, 1977. 144p. Irrigation and Dreanaige Paper, 24

Eastman, J. R. IDRISI32 - Clark Unversity - The IDRISI project. Worcester: Clark Labs, 1999. 557p.

Fancelli, A. L.; Dourado Neto, D. Produção de milho. Guaíba: Agropecuária, 2000. 360p.
Faria, R. A.; Soares, A. A.; Sediyama, G. C.; Ribeiro, C. A. A. S. Economia de água e energia em projetos de irrigação suplementar no Estado de Minas Gerais. Revista Brasileira de Engenharia Agrícola e Ambiental, Campina Grande, v.6, n.2, p.189-194, 2002.

Jensen, M. E.; Burman, R. D.; Allen, R. G. Evapotranspiration and irrigation water requiriments. New York: ASCE, 1990, 332p.

Kashyap, P. S.; Panda, R. K. Evaluation of evapotranspiration estimation methods and development of crop-coefficients for potato crop in a sub-humid region. Agricultural Water Management. Amsterdam. v.50, n.1. p.9-25. 2001.

Lima, M. G.; Costa, E. F.; Gonzales, H. L. Demanda de água pela cultura do milho híbrido XL-560. In: Seminário de pesquisa agropecuária O Piauí, 6, 1990. Teresina. Anais... Teresina: Embrapa-UEPAE, 1992. p.207-215.

Pereira, L. S.; Allen, R. G. Novas aproximações aos coeficientes culturais. Engenharia Agrícola, Jaboticabal, v.16, n.4, p.118143. 1997.

Resende, M.; França, G. E.; Couto, L. Cultivo do milho irrigado. Sete Lagoas: Embrapa Milho e Sorgo, 2000. 39p. Circular Técnica 6

Saad J. C. C.; Frizzone, J. A. Estudo da distribuição de freqüência da precipitação pluvial visando ao dimensionamento de sistemas de irrigação. Irriga, Botucatu, v.3 n.2. p.35-46, 1998.

Sediyama, G. C.; Melo, J. S. P.; Alves, A. R.; Coelho, D. T. Determinação dos parâmetros da distribuição gama, em função das alturas mensais de precipitação dos dias chuvosos. Revista Ceres, Viçosa, v.43, n.274, p.254-266, 1996.

Smith, M. Report on the expert consultation on revision of FAO methodologies for crop water requirements. Rome: FAO, 1991. 45p.

Sousa, E. F.; Bernardo, S.; Azevedo, H. J.; Berbert, P. A.; Viana, A. P. Estimativa da demanda hídrica provável na região Norte Fluminense. Revista Engenharia Agrícola, Jaboticabal, v.22, n.3, p.322-331, 2002.

Souza Filho, B. F.; Fernandes, G. M. B.; Yujra, P. R. R. Cultivo de feijão em várzeas em sucessão ao arroz. Niterói: Pesagro Rio, 1987. 4p. Comunicado Técnico, n.179 